\title{
Synthesis of 3-Fluoropyrazoles from 2-Trifluoromethyl-1-alkenes
}

Key words

3-fluoropyrazoles

hydrazines

trifluoromethylstyrenes
Significance: Reported is a three-step protocol for the de novo synthesis of substituted 3-fluoropyrazoles through annulation of 2-trifluoromethyl1 -alkenes with monosubstituted hydrazines. The first step in this unconventional approach is an $\mathrm{S}_{\mathrm{N}} 2^{\prime}$ addition of an $\mathrm{N}$-deprotonated hydrazine to the trifluoromethyl-substituted alkene to give a 3,3-difluoro allylic hydrazide, which is subsequently tosylated $(\mathbf{1} \rightarrow \mathbf{2})$. While $\mathrm{N}$-alkylation proceeds in a highly regioselective manner when aryl- and Bocsubstituted hydrazines are employed, methylhydrazine affords a 55:45 mixture of $\mathrm{N}$-regioisomers (66\% combined yield, not shown above). Treatment of $\mathbf{2}$ with $\mathrm{NaH}$ in DMF affords the substituted 3-fluoropyrazole 3; control experiments established the need to employ tosylhydrazides in this reaction. 4-Unsubstituted 3-fluoropyrazoles 5 were accessible from the corresponding 2-silyl allylic hydrazide 4

SYNFACTS Contributors: Victor Snieckus, Matthew S. Dowling (Pfizer) Synfacts 2013, 9(1), $0021 \quad$ Published online: 17.12.2012 Dol: 10.1055/s-0032-1317874; Reg-No.: V15812SF
Comment: Pyrazoles are among the most metabolically stable unsaturated five-membered heterocycles (see Review below) and are frequently incorporated into drug candidates. A successful example is the COX-2 inhibitor celebrex ${ }^{\circledR}$. The present method provides efficient access to synthetically challenging substituted 3-fluoropyrazoles through a non-obvious and generally highyielding annulation sequence that utilizes readily accessible starting materials. On the down side, no mention was made of attempts to achieve the synthesis of C5-substituted pyrazoles; alkyl substitution at $\mathrm{C} 4$ was also not explored. Control experiments suggest that base-mediated ring closure $(\mathbf{2} \rightarrow \mathbf{3})$ proceeds through neither direct nucleophilic vinylic substitution $\left(\mathrm{S}_{N} \mathrm{~V}\right)$ nor an intermediate nitrene. Instead, an unusual pathway is suggested that features an azomethine imine intermediate.

Review: D. K. Dalvie et al. Chem. Res. Toxicol. 2002, 15, 269-299. 